\title{
Collection, spillback, and dissipation in pedestrian evacuation: a network-based method
}

\author{
Ren-Yong Guo ${ }^{a}$, Hai-Jun Huang ${ }^{b^{*}}$, S.C. Wong ${ }^{c}$ \\ ${ }^{a}$ College of Computer Science, Inner Mongolia University, Hohhot 010021, China \\ ${ }^{\mathrm{b}}$ School of Economics and Management, Beijing University of Aeronautics and Astronautics, Beijing \\ 100191, China
}

${ }^{\mathrm{c}}$ Department of Civil Engineering, The University of Hong Kong, Pokfulam Road, Hong Kong, China

\begin{abstract}
We present a method of predicting pedestrian route choice behavior and physical congestion during the evacuation of indoor areas with internal obstacles. Under the proposed method, a network is first constructed by discretizing the space into regular hexagonal cells and giving these cells potentials before a modified cell transmission model is employed to predict the evolution of pedestrian flow in the network over time and space. Several properties of this cell transmission model are explored. The method can be used to predict the evolution of pedestrian flow over time and space in indoor areas with internal obstacles and to investigate the collection, spillback, and dissipation behavior of pedestrians passing through a bottleneck. The cell transmission model is further extended to imitate the movements of multiple flows of pedestrians with different destinations. An algorithm based on generalized cell potential is also developed to assign the pedestrian flow.
\end{abstract}

Keywords: pedestrian evacuation; evolutionary network; collection; spillback; dissipation

\section{Introduction}

Walking is a trip mode involved in most daily human activities such as crossing the road, shopping, and coming together for group activities. With population increases and rapid urbanization, there is increasing pressure on existing urban roads and the energy resources

\footnotetext{
${ }^{*}$ Corresponding author. Tel.: +86-10-82316608; fax: +86-10-82328037.

E-mail address: haijunhuang@buaa.edu.cn
} 
required for vehicular transport are becoming scarcer. As a result, walking is coming to play an increasingly important role in the development of successful sustainable transportation (Cervero et al., 2009; Walton and Sunseri, 2010). Research on pedestrians has recently attracted a significant level of interest in transport-planning societies.

Pedestrian evacuation is a strategy commonly used to handle emergency situations. If the internal condition of a building evacuated in an emergency is not suitable for the evacuation process or if pedestrian behavior cannot be controlled or guided effectively, then the ramifications of the emergency situation cannot be alleviated and additional losses are likely to ensue. Thus, it is essential that formal scientific analysis of the pedestrian evacuation process, and of pedestrian evacuation behavior in particular, be carried out to reduce and avoid losses in emergency situations.

Several computer models have been developed to simulate the evacuation patterns of pedestrians and predict evacuation performance with a view to generating valuable information for the assessment, evaluation, and design of building evacuation systems. These models can generally be divided into two categories according to mode of implementation.

All network-based models can be placed in the first category (e.g., Cepolina , 2005, 2009; Asano et al., 2007; Lin et al., 2008; Chen and Feng, 2009; Yuan et al., 2009; Pursals and Garzón, 2009). In this class of models, the spatial layout of a building is represented by a network based on the building's actual structure. Accordingly, each node in the network may represent a room, lobby, or section of corridor irrespective of its physical dimensions. These nodes are connected by arcs that represent the actual openings between separate components. This class of models is generally used to calculate evacuation plan performance measures. Another application of these models is in the formulation of solutions to optimization problems. Solutions to these problems aim to minimize the evacuation time of pedestrians leaving the building last or the total time taken to evacuate all pedestrians from a building, or to maximize the number of pedestrians leaving the building in a certain time interval.

The second category comprises the area-based models. These models simulate pedestrian dynamics in closed areas such as rooms, corridors, and supermarkets. In this class of models, either a crowd is regarded as a fluid or continuum that responds to local influences (e.g., 
Thompson and Marchant, 1995; Hughes, 2002; Xia et al., 2008, 2009; Huang et al., 2009) or pedestrians are treated as discrete individuals (e.g., Helbing and Molnár, 1995; Helbing et al., 2000; Burstedde et al., 2001; Kirchner and Schadschneider, 2002; Langston et al., 2006; Guo et al., 2010). There is no doubt that the area-based models give more accurate predictions and more detailed information on evacuation processes than the network-based models. However, this class of models is unsuitable for tall, large, or complex buildings due to their complex internal construction and the large amount of computing time required.

Both categories of models involve two tasks that are relatively difficult to accomplish. The first is the construction of a plausible framework for pedestrian movement route choice behavior when pedestrians attempt to leave closed areas with internal obstacles. The second is the formulation of physical congestion and interaction among pedestrians. The purpose of this paper is to design and develop a method that reproduces the pedestrian evacuation process in areas with internal obstacles and predicts the collection, spillback, and dissipation behavior of pedestrians near exits or obstacles. This work is challenging because the two tasks must be resolved simultaneously, it is difficult to generate a good space representation in the presence of internal obstacles, and pedestrian movement is flexible in two-dimensional space.

In the proposed method, the pedestrian evacuation space is represented by a network using the so-called potential distribution of the space. A number of existing dynamic traffic assignment methods can then be applied directly to solve the pedestrian evacuation problem. In this paper, a cell transmission model is devised to formulate the movements of the flow of pedestrians in the network. Simulation results indicate that our method can mimic the pedestrian evacuation process in areas with internal obstacles and can model the collection, spillback, and dissipation behavior of pedestrians near exits. Furthermore, the cell transmission model is extended to formulate the collection, dissipation, and propagation behavior of multiple flows of pedestrians targeting different exits in a closed area with multiple exits during evacuation. An algorithm based on generalized cell potential that considers both the route distance to the destination and the congestion of pedestrians in front routes is also developed to assign pedestrian flow in space.

The remainder of this paper is organized as follows. Section 2 introduces the method 
proposed for converting the pedestrian evacuation problem into a network problem and the flow propagation model developed to formulate pedestrian movement. In this section, we also introduce the method used to predict the evolution of pedestrian flow over time and space in indoor areas with internal obstacles and to model the collection, spillback, and dissipation behavior of pedestrians passing through bottlenecks. In Section 3, a modified cell transmission model is proposed to formulate the movement of multiple flows of pedestrians targeting different exits. Section 4 describes the algorithm developed on the basis of generalized cell potential. Section 5 concludes the paper. The properties of the propagation model are presented in the Appendix.

\section{Method}

\subsection{Network representation of the pedestrian evacuation problem}

Consider an indoor area with internal obstacles such as furniture, shelves, devices, and electrical appliances. Regardless of the size and position of the obstacles, the entire space in the indoor area is discretized into regular hexagonal cells with a side length $s$ (see Fig. 1). The cell structure is isotropic, allowing for the pedestrian flow into neighboring cells to be defined in identical terms. If square cells were adopted, formulating the pedestrian flow into diagonal neighboring cells would become difficult. Each cell can accommodate a certain maximum number of pedestrians in proportion to the area of free space in the cell, and pedestrians in each cell may move into six neighboring cells. The states of the pedestrians are updated at each time interval $\Delta t$. Note that the distance $d \quad(=\sqrt{3} s)$ between the centers of two cells is set so that $d=v_{0} \Delta t$, where $v_{0}$ is the free-flow velocity of the pedestrians.

Non-connective and connective cells are defined. If there are obstacles at the boundary between two neighboring cells and pedestrian movement between these two cells is completely blocked, then the two cells are referred to as non-connective cells. Fig. 1 illustrates a pedestrian space with three cells and an obstacle. Cells 1 and 3 are non-connective and cells 1 and 2 are connective. 


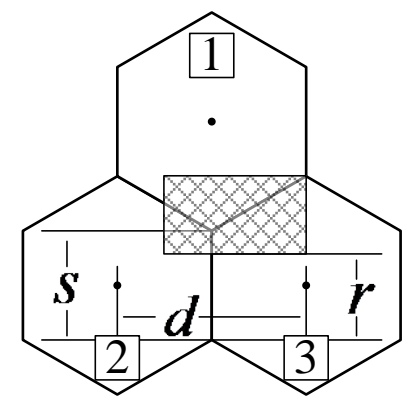

Fig. 1. A pedestrian space with three cells (the shaded rectangle denotes an obstacle).

The potential of each cell is defined and used to approximate the route distance to the target exit and to reflect the number of minimal movements to the target exit (Huang and Guo, 2008; Guo and Huang, 2010). The potential increases with the distance to the exit. It is assumed that pedestrians select target cells at the next time step to reduce the potential to their destination at each time step. This means that pedestrians consider the route distance to each exit in selecting their movement routes. Empirical evidence indicates that although pedestrians frequently choose the shortest route, they are seldom aware that they are minimizing the distance as a primary strategy in route choice (Senevarante and Morall, 1985; Hoogendoorn and Bovy, 2004a). It is sufficient for a pedestrian model based on this assumption to provide valuable information for designing and adjusting the internal layout and exit position of buildings.

Let $C$ be the set of cells in the entire pedestrian space, $C^{e}$ the set of cells near exit $e$, $C^{n}$ the set of cells that are non-connective with all six neighboring cells, and $P_{i}^{e}$ the potential of cell $i$ corresponding to exit $e$. The potentials of the cells are computed by the following algorithm.

Step 1: For all cells $i \in C \backslash C^{e}$, set $\bar{P}_{i}^{e}=0$; for all cells $j \in C^{e}$, set $\bar{P}_{j}^{e}=1$. Set $l=1$;

Step 2: For each cell $i \in C$ with $\bar{P}_{i}^{e}=l$, check its adjacent cell $j$ in all six directions. If $\bar{P}_{j}^{e}=0$ and cells $i$ and $j$ are connective, then let $\bar{P}_{j}^{e}=l+1$;

Step 3: If $\bar{P}_{i}^{e}>0$ holds for any one cell $i \in C \backslash C^{n}$, then go to step 4; otherwise set $l \leftarrow l+1$ and go to step 2;

Step 4: For each cell $i \in C$ with $\bar{P}_{i}^{e}=1$, set $P_{i}^{e}=1$. Set $l=2$; 
Step 5: For each cell $i \in C$ with $\bar{P}_{i}^{e}=l$, check its adjacent cells in all six directions. If $\left|\Omega_{i}^{e}\right|=1 \quad$ and $\quad j \in \Omega_{i}^{e}$, then set $P_{i}^{e}=P_{j}^{e}+1 ; \quad$ if $\quad\left|\Omega_{i}^{e}\right|>1$, then set $P_{i}^{e}=\sum_{j \in \Omega_{i}^{e}} P_{j}^{e} /\left|\Omega_{i}^{e}\right|+\theta$. Here, $\quad \Omega_{i}^{e}=\left\{j \mid\right.$ cells $j$ and $i$ are connective, and $\left.\bar{P}_{j}^{e}=l-1\right\}$ and $\theta \in(0,1]$;

Step 6: If $l=\max \left\{\bar{P}_{i}^{e} \mid i \in C\right\}$, stop; otherwise set $l \leftarrow l+1$ and go to step 5 .

In the foregoing computation, parameter $\bar{P}_{i}^{e}$ measures the number of minimal movements along the cells to exit $e$. It is used to determine the sequence in which the potentials of cells are obtained. The potential of a cell is computed using the potentials of one or more cells for which the potentials were computed in the previous step. To approximate the route distance from a cell to the target exit according to the potential of the cell with a higher degree of connectivity, the potential of the cell is computed in two cases. These cases are differentiated by the number of cells for which the potentials were computed in the previous step. If the number is one, that is, $\left|\Omega_{i}^{e}\right|=1$, the potential of the cell increases for one; if the number is more than one, that is, $\left|\Omega_{i}^{e}\right|>1$, the potential of the cell increases for a $\theta$ value of not more than one. The value of the parameter $\theta$ can be calibrated by using the least square method so that the potentials of the cells approximate the route distance to each target exit from cells with a higher degree of connectivity. In addition, this parameter can also be calibrated by formulating the shape of the crowd near an exit. The shape of the crowd is approximately semicircular, reflecting a commonly observed scenario (see numerical simulations in Section 2.3).

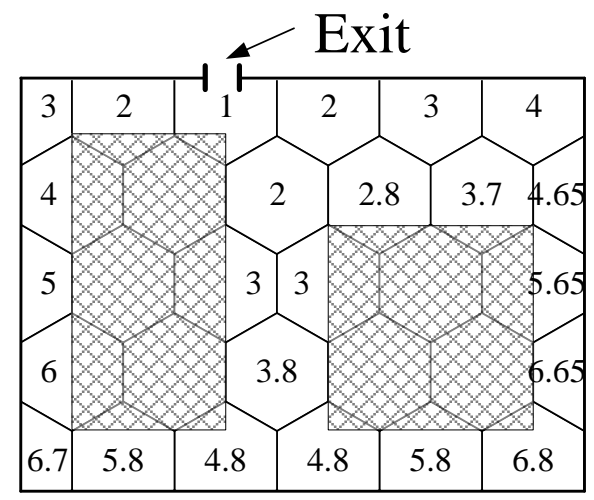

Fig. 2. A room with one exit and two internal obstacles denoted by shaded rectangles and the 
potentials of all cells corresponding to the exit when $\theta=0.8$. The number in each cell is the potential of the cell.

Fig. 2 gives the potentials of all of the cells in a room with one exit and two internal obstacles when $\theta=0.8$. It can be seen that the cells closest to the exit have a lower potential. Thus, their potential can be used to determine pedestrian routes during evacuation.

Once the potentials of the cells have been determined, the cells in set $C \backslash C^{n}$ can be regarded as nodes in a network. Each pair of connective cells are linked by a directed arc in which the cell with the larger potential is at one end and the cell with the smaller potential is at the other end. The direction of the arc indicates the movement of pedestrians from a cell with a larger potential to a connective cell with a smaller potential. All nodes corresponding to cells near an exit are linked to a virtual destination node. In this way, the pedestrian space is converted into a network. For instance, the room in Fig. 2 can be represented by the network shown in Fig. 3.

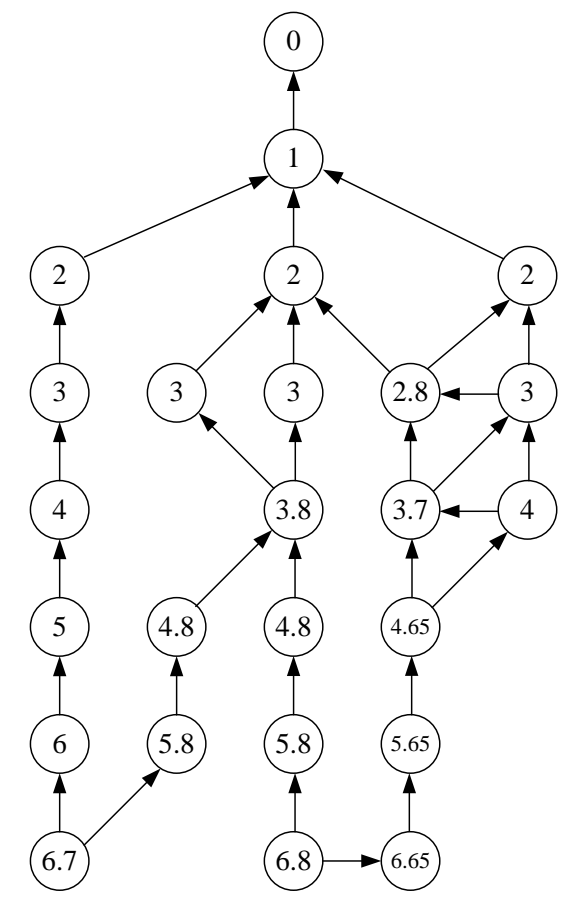

Fig. 3. Network representation of the room shown in Fig. 2. The number in each node is the potential of the corresponding cell and the node with a potential of 0 is a virtual destination node. 
Using this network representation, some existing models and solution methods developed for the study of static or dynamic vehicle traffic assignment (e.g., Huang and Lam, 2002; Lo and Szeto, 2002; Szeto and Lo, 2004, 2006) can be applied directly to pedestrian evacuation problems. The cell transmission model developed by Daganzo $(1994,1995)$ has been used to load traffic flow on dynamic networks and has been shown to be capable of capturing traffic dynamics such as shockwaves, queue formation, and queue dissipation. The following section proposes a cell transmission model for pedestrian movement that captures the pedestrian dynamics in the aforementioned network.

\subsection{Pedestrian propagation model}

A cell transmission model is proposed to formulate pedestrian propagation in indoor areas. First, the maximum flows of the arcs and the capacities of the nodes in the evacuation network must be defined. Let $Q_{i j}(t)$ denote the maximum number of pedestrians who move to node $j$ from node $i$ in interval $\Delta t$ between time steps $t$ and $t+1$. For a destination node $j_{0}$ and the upstream linked node $i_{0}$, the maximum number $Q_{i_{0} j_{0}}(t)$ is regarded as the number of pedestrians passing through the exit in interval $\Delta t$ between time steps $t$ and $t+1$. For two nodes $i$ and $j$, which correspond to two neighboring cells, it is assumed that the maximum number $Q_{i j}(t)$ is proportional to the size $r_{i j}(t)$ of the opening available between the two cells in interval $\Delta t$ between time steps $t$ and $t+1$, that is,

$$
Q_{i j}(t)=\frac{r_{i j}(t)}{s} \bar{Q},
$$

where $s$ is the side length of the regular hexagonal cells and $\bar{Q}$ is the maximum number of pedestrians exiting through the opening between the two cells in interval $\Delta t$. The size of the opening available between two cells is illustrated in Fig. 1 . For instance, $r_{23}=r$ for cells 2 and 3 in the figure, whereas $r_{13}=0$ for cells 1 and 3 .

Let $N_{i}(t)$ denote the maximum number of pedestrians present at node $i$ at time step $t$. For each destination node $i_{0}$, set $N_{i_{0}}(t)=+\infty$. For node $i$, which corresponds to a cell, the maximum number $N_{i}(t)$ is proportional to the area $a_{i}(t)$ of the space in the cell 
unoccupied by obstacles at time step $t$, or

$$
N_{i}(t)=\frac{a_{i}(t)}{c} \bar{N},
$$

where $c=(3 \sqrt{3} / 2) s^{2}$ is the area of the cell and $\bar{N}$ is the maximum capacity of an empty cell to accommodate pedestrians.

Several other variables are used in the propagation model. Let $n_{i}(t)$ be the number of pedestrians at node $i$ at time step $t$ and let $D_{i j}(t)$ be the proportion of pedestrians at node $i$ who can move to the adjacent downstream node $j$ in interval $\Delta t$ between time steps $t$ and $t+1$. It is assumed that $D_{i j}(t)$ is positively proportional to the product of the number of pedestrians accommodated by the empty space at node $j$ at time step $t, N_{j}(t)-n_{j}(t)$, and the difference between the potentials of the two cells corresponding to nodes $i$ and $j$, $P_{i}-P_{j}$. We therefore have

$$
D_{i j}(t)=\frac{\left(P_{i}-P_{j}\right)\left[N_{j}(t)-n_{j}(t)\right]}{\sum_{k \in R_{i}}\left\{\left(P_{i}-P_{k}\right)\left[N_{k}(t)-n_{k}(t)\right]\right\}},
$$

where $R_{i}$ is the set of neighboring nodes downstream of node $i$. Hill (1982) concludes that like most walking processes, the route selection strategies of pedestrians are largely subconscious. Our model assumes that pedestrians select target nodes at the next time step by considering not only the movement distance, but also by taking into account the degree of local congestion in the front nodes. Let $w_{i j}(t)$ be the number of pedestrians who can be sent to node $j$ from the adjacent upstream node $i$ in interval $\Delta t$ between time steps $t$ and $t+1$. This gives

$$
w_{i j}(t)=\min \left\{D_{i j}(t) n_{i}(t), Q_{i j}(t)\right\} .
$$

It is unlikely that the empty space at a node can accommodate all of the pedestrians can be sent to the node from adjacent upstream nodes. It is therefore assumed that the proportion of empty space at a node that can receive pedestrians from an adjacent upstream node is partly related to the number of pedestrians who can be sent to the node from upstream nodes. The number of pedestrians who move from node $i$ to the adjacent downstream node $j$ in interval $\Delta t$ between time steps $t$ and $t+1, y_{i j}(t)$, is then given by 


$$
y_{i j}(t)= \begin{cases}w_{i j}(t), & \sum_{k \in S_{j}} w_{k j}(t) \leq N_{j}(t)-n_{j}(t), \\ \frac{N_{j}(t)-n_{j}(t)}{\sum_{k \in S_{j}} w_{k j}(t)} w_{i j}(t), & \text { otherwise, }\end{cases}
$$

where $S_{j}$ is the set of neighboring nodes upstream of node $j$. Eq. (5) can be rewritten as

$$
y_{i j}(t)=\min \left\{w_{i j}(t), \frac{N_{j}(t)-n_{j}(t)}{\sum_{k \in S_{j}} w_{k j}(t)} w_{i j}(t)\right\} .
$$

Based on the foregoing definitions, the propagation of pedestrian flow in the evacuation network can be described by the following equation for all nodes and time steps.

$$
n_{i}(t+1)=n_{i}(t)+\sum_{k \in S_{i}} y_{k i}(t)-\sum_{j \in R_{i}} y_{i j}(t) .
$$

Several properties of the above propagation model are provided in the Appendix.

\subsection{Numerical Simulations}

The proposed modeling approach is applied to the two simulation scenarios shown in Fig. 4 (a) and (b). These two scenarios are the same in terms of size and exits, but have different internal configurations. There are no obstacles in scenario (a), whereas there are six obstacles in scenario (b). For both scenarios, the indoor areas are discretized into $19 \times 6+20 \times 5$ cells and an exit is placed in the north wall. The following parameters are used in the experiments: the time interval $\Delta t=1.2$, the width of the cells $s=1.0$, the maximum number of pedestrians passing through the opening between two cells in a time interval $\bar{Q}=10$, the maximum capacity of an empty cell to accommodate pedestrians $\bar{N}=16$, and the maximum number of pedestrians moving from an upstream linked node $i_{0}$ to a destination node $j_{0}$ in each time interval (i.e., the maximum number of pedestrians passing through the exit in one time interval) $Q_{i_{0} j_{0}}(t)=15$.

One of the most remarkable characteristics of animal groups such as flocks of birds, schools of fish, and swarms of locusts is how they behave in forming such groups (e.g., Parrish and Edelstein-Keshet, 1999; Couzin et al., 2002, 2005; Cucker and Smale, 2007). Some types of collective behavior exhibited by animal groups can be regarded as the result of 
competition among group members for limited resources. When a large number of pedestrians pass through a bottleneck together, an irregular succession of arch-like crowds can be observed near the bottleneck. This type of collective behavior has been reproduced in microscopic pedestrian models such as the social force model (Helbing et al., 2000) and the cellular automata model (Kirchner and Schadschneider, 2002). We use our proposed method to investigate the collective behavior of pedestrians near exits during evacuation.
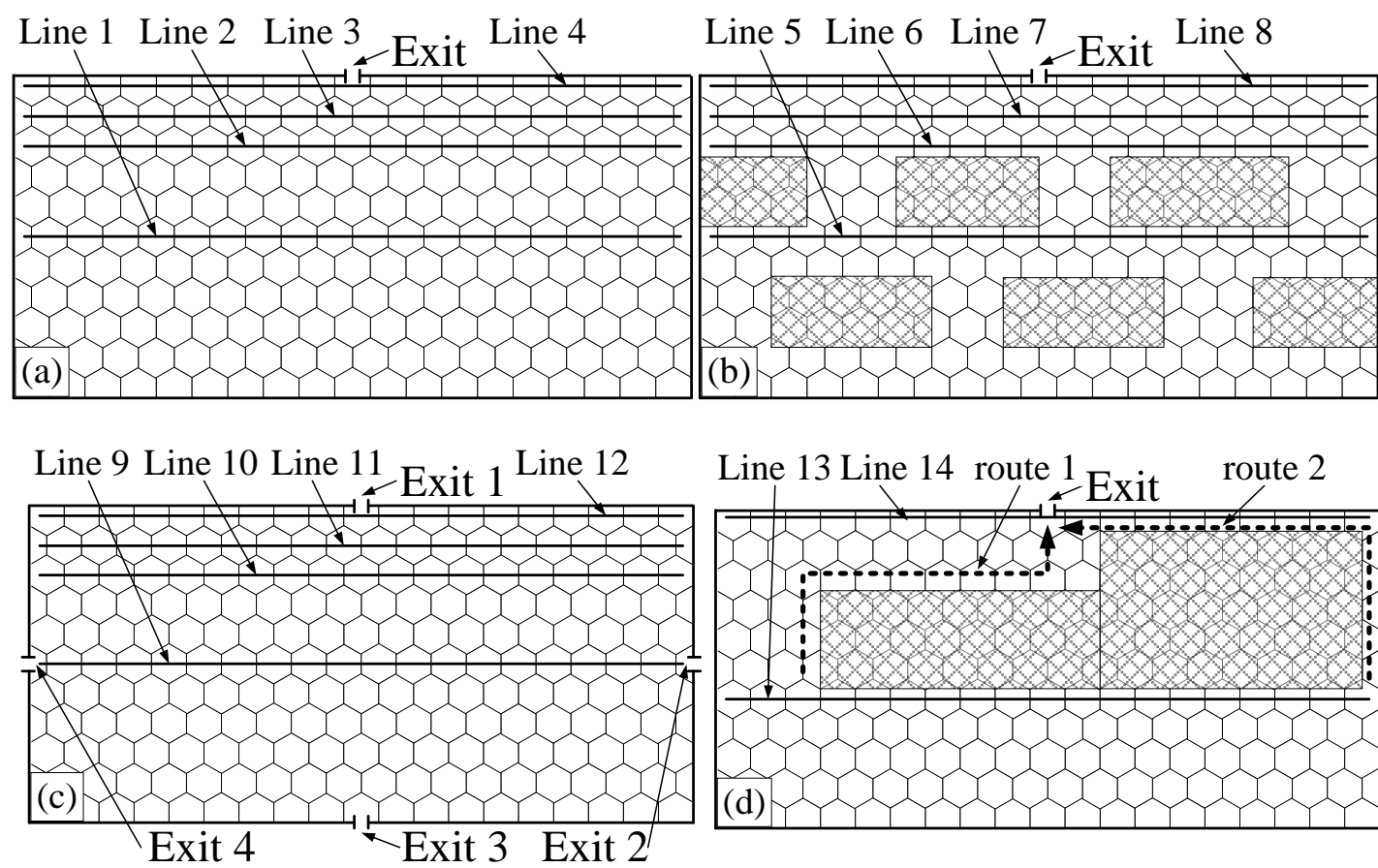

Fig. 4. Four simulation scenarios identical in size but with different exits and internal configurations. For all scenarios, the indoor areas are discretized into $19 \times 6+20 \times 5$ cells. (a) An exit in the north wall and no obstacles in the indoor area. (b) An exit in the north wall and six obstacles denoted by the shaded rectangles in the indoor area. (c) Four exits and no obstacles in the indoor area. (d) An exit in the north wall and two obstacles in the indoor area.

Fig. 5 shows pseudo-color plots delineating the ratio of the number of pedestrians in each cell to the capacity of the cell in scenario (a) at time steps 10, 40, and 80 in the simulation. The ratio of the number of pedestrians in each cell to the capacity of the cell at the initial time is 0.35 , and the parameter $\theta$ in the algorithm used to compute the potential of each cell takes the values of $0.5,0.7,0.9,0.95$, and 1.0, respectively. Although our 
network-based model needs less computation time than most microscopic pedestrian models, the appearance and disappearance of arching crowds near the exit is clearly discernible in the figure for each $\theta$ value.
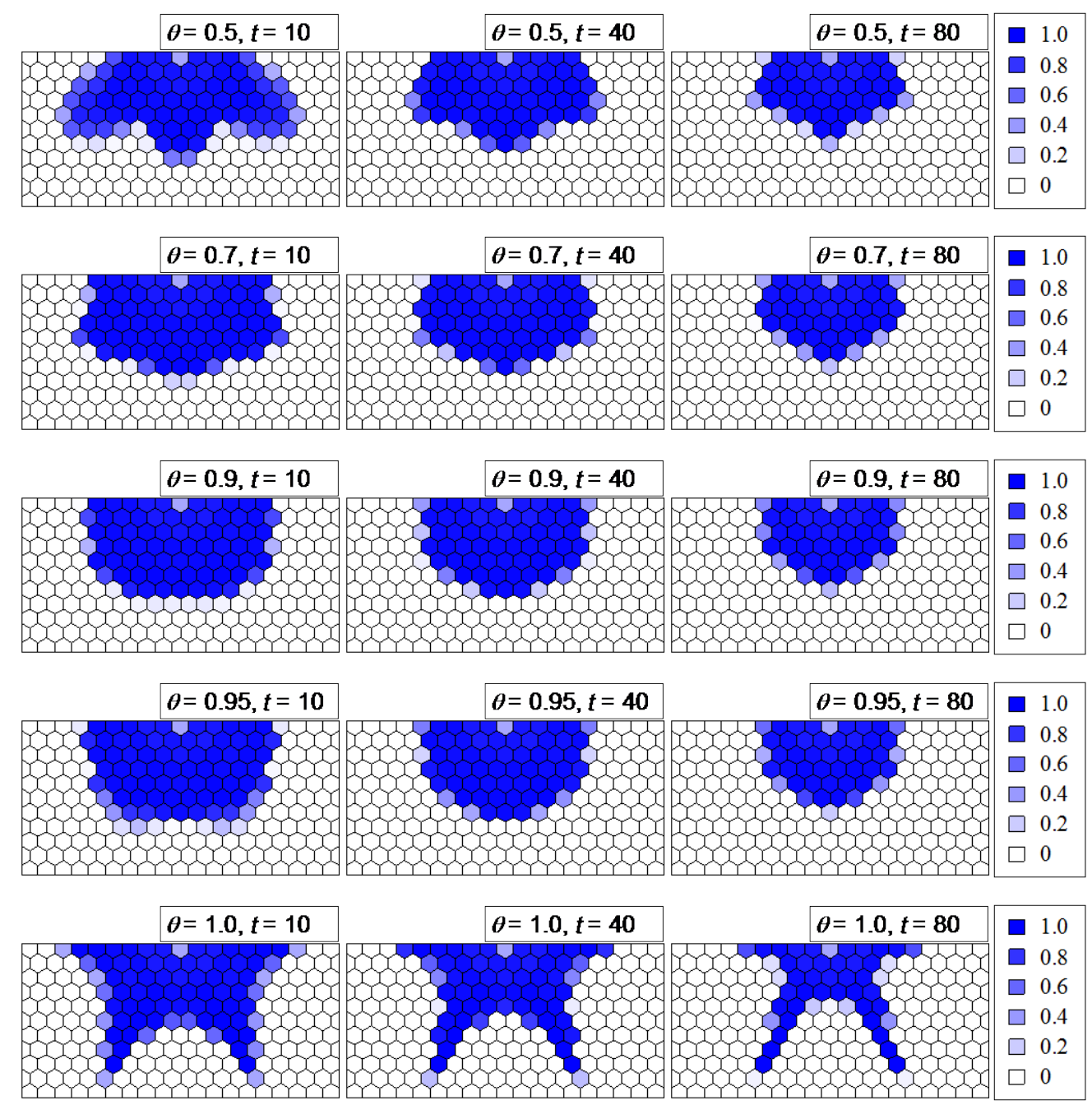

Fig. 5. Pseudo-color plots delineating the ratio of the number of pedestrians in each cell to the capacity of the cell at time steps 10, 40, and 80 during an evacuation process in scenario (a) when $n_{i}(0)=0.35 N_{i}(0)$ (where $i$ is any node) and the parameter $\theta=0.5,0.7,0.9,0.95$, and 1.0.

It can also be seen that when $\theta=0.5$, the vertical and diagonal dimensions of the crowd are relatively large and the horizontal dimension is relatively small. As the $\theta$ value increases, the vertical and diagonal dimensions of the crowd decrease and the horizontal dimension increases. When $\theta=0.9$ and 0.95 , the shape of the crowd is approximately semicircular, 
reflecting a commonly observed scenario. When the $\theta$ value increases to 1.0 , an unreasonable crowd shape with larger horizontal and oblique dimensions and a smaller vertical dimension emerges. When $\theta=1$, the potentials of the cells are equal to the number of minimal movements along the cells to the exits. Among cells equally distant from an exit, those in the horizontal and oblique directions have smaller potential. As a result, most pedestrians gather in the horizontal and oblique directions and an odd-shaped crowd forms. Thus, it is suggested that the $\theta$ value should be within the range $[0.9,0.99]$.

We now investigate the collective behavior of pedestrians near the exit in an indoor area with internal obstacles during evacuation. Fig. 6 displays pseudo-color plots delineating the ratio of the number of pedestrians in each cell to the capacity of the cell in scenario (b) at time steps $5,20,40,60,80$, and 100 during an evacuation process. The number of pedestrians at each node at the initial time step is 0.35 of the capacity and the parameter $\theta$ takes the value of 0.9 . The figure shows that in the process of evacuation, pedestrians bypass these obstacles to move forward to the exit and collective behavior occurs near the exit. This indicates that our method can be applied to model the evacuation of pedestrians from indoor areas with internal obstacles.

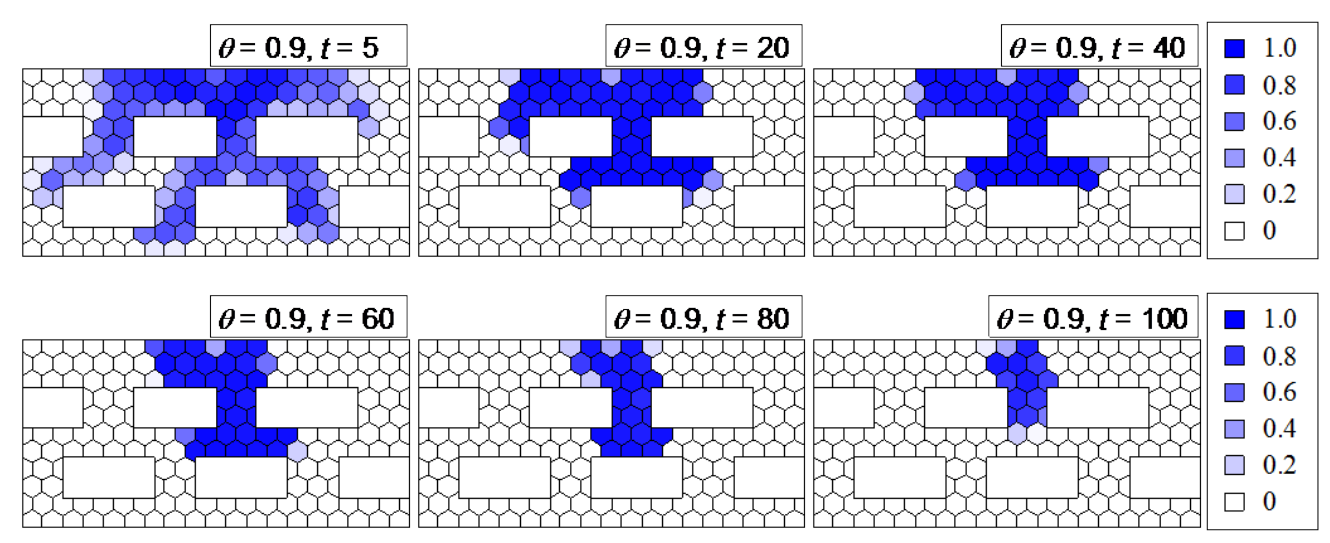

Fig. 6. Pseudo-color plots delineating the ratio of the number of pedestrians in each cell to the capacity of the cell at time steps 5, 20, 40, 60, 80, and 100 during evacuation in scenario (b) when $n_{i}(0)=0.35 N_{i}(0)$ (where $i$ is any node) and $\theta=0.9$.

It can also be seen that two of the obstacles close to the exit block the oblique paths to 
the exit and that pedestrians approaching the exit can move along the horizontal and vertical paths only. Because the number of pedestrians accumulating on the vertical paths is greater than the number of pedestrians coming together on the horizontal paths, the pedestrians on the vertical paths take more time to leave the area due to congestion. Pedestrians gather in the cells near the exit along the horizontal and vertical paths simultaneously before a certain time step. After that time step, pedestrians on the horizontal paths have already left the indoor area, whereas those on the vertical paths have not. This means that the specific bottleneck space in the room has been identified.
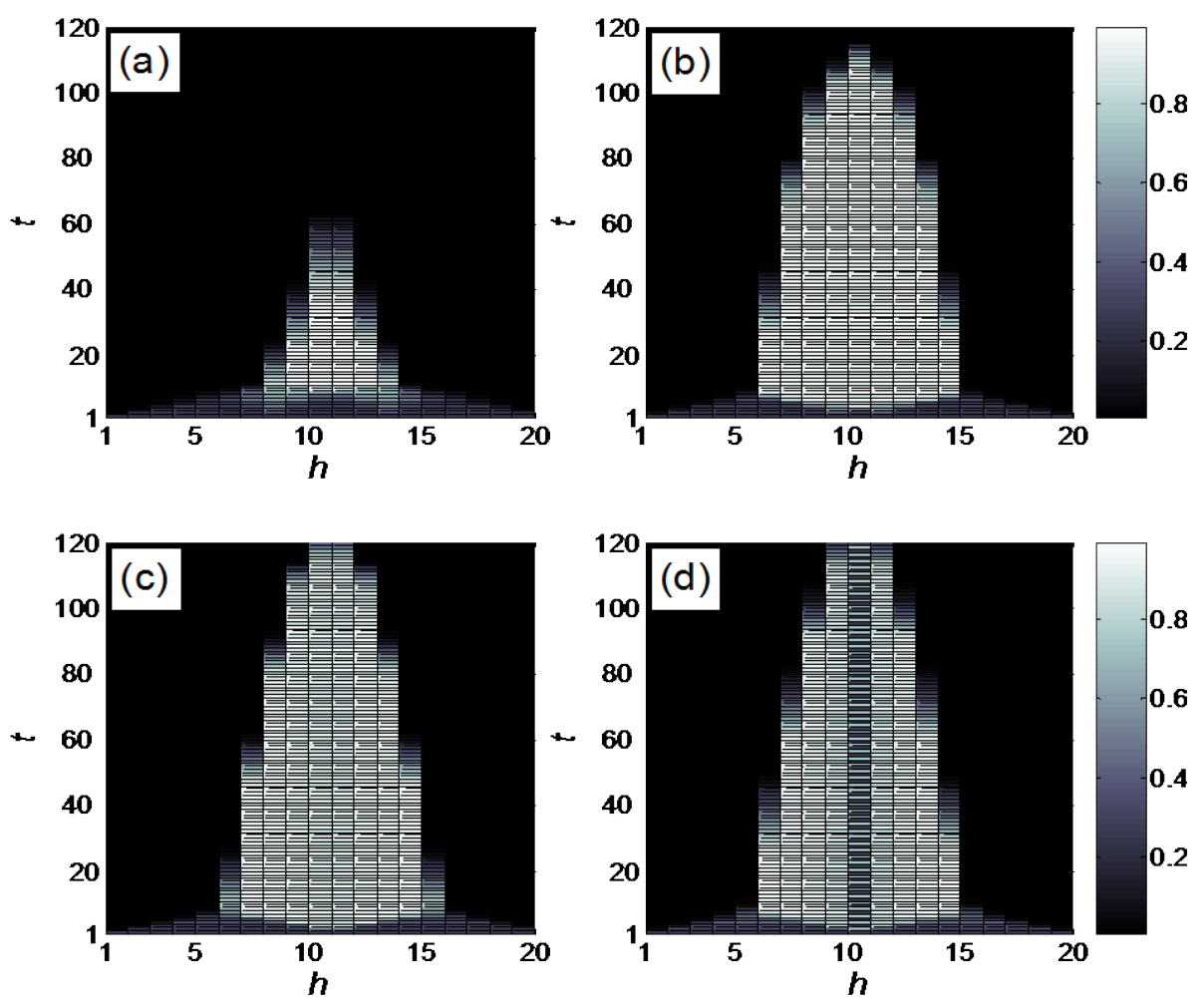

Fig. 7. Time-space diagrams delineating the ratio of the number of pedestrians in each cell to the capacity of the cell on lines (a) 1, (b) 2, (c) 3, and (d) 4 in Fig. 4 when $\theta=0.9$ and $n_{i}(0)=0.25 N_{i}(0)$ (where $i$ is any node). $h$ denotes the horizontal coordinates of the cells on lines 1-4.

We now investigate spillback and dissipation among pedestrians on lines 1-8 (see Fig. 4) as the final part of the initial analysis. At each time step, pedestrians not only move along the 
queues on each line, but also move into or out of the queues. The spillback and dissipation behavior of these pedestrians is different from that of vehicle traffic on a single lane. Figs. 7 (a)-(d) show time-space diagrams delineating the ratio of the number of pedestrians in each cell to the capacity of the cell on lines 1-4 when $\theta=0.9$ and $n_{i}(0)=0.25 N_{i}(0)$ (where $i$ is any node). It can be seen that the evolution of the pedestrian flow on these lines over time is divided into three phases. In phase one, the ends of the free flow in the cells relatively distant from the exit dissipate and the congested flow in the cells relatively close to the exit propagates to both ends of the lines. As a result, the ends of the pedestrian queues move to the middle of the lines and pedestrians come together in the corresponding portions of the cells. In phase two, the cells occupied by pedestrians are saturated and the congested flow in these cells remains unchanged for some time. In phase three, the congested flow in the cell at the end of each queue eases and the length of the pedestrian queue decreases. Once phase one has occurred, phases two and three alternate until all of the pedestrians on these lines have dissipated.

The queues on the lines close to the exit require more time to dissipate. In phase one, the congested flow on line 1 , which is relatively distant from the exit, propagates to the two ends of the line at two positions deviating from the middle of the line, whereas the congested flow in each of lines 2-4, which are relatively close to the exit, propagates at one position in the middle of the line.

Figs. 8 (a)-(d) are time-space diagrams delineating the ratio of the number of pedestrians in each cell to the capacity of the cell in lines 5-8 when $\theta=0.9$ and $n_{i}(0)=0.25 N_{i}(0) \quad(i$ is any node). The three phases already described can again be observed. However, there are two main differences between Figs. 7 and 8. On lines 5 and 6, which are relatively close to obstacles, the dissipation of the ends of the free flow and the propagation of the congested flow in phase one are irregular due to the obstacles. The time required to empty the cells on either side of the middle of these lines is different. The time required to empty the cell on the left-hand side is less than that required to empty the corresponding cell on the right-hand side due to the existence of obstacles and the geometry of the space. 

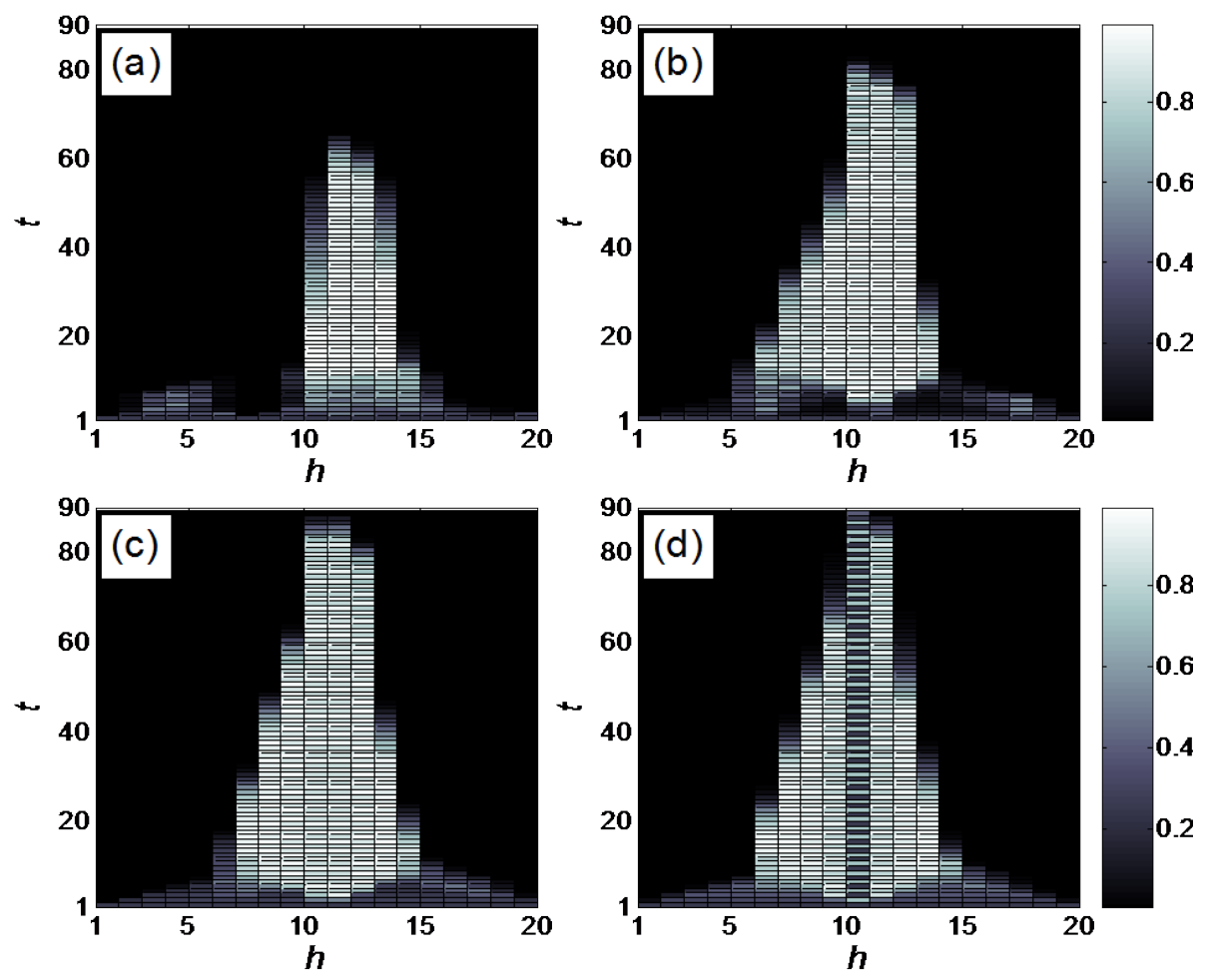

Fig. 8. Time-space diagrams delineating the ratio of the number of pedestrians in each cell to the capacity of the cell on lines (a) 5, (b) 6, (c) 7, and (d) 8 in Fig. 4 when $\theta=0.9$ and $n_{i}(0)=0.25 N_{i}(0)$ ( $i$ is any node). $h$ denotes the horizontal coordinates of the cells on lines 5-8.

\section{Propagation model for multiple flows of pedestrians}

\subsection{Model description}

The cell transmission model proposed in the previous section considers a flow of pedestrians with one destination, i.e., the model is used to predict the propagation of pedestrians in indoor areas with only one exit. In this section, the model is extended to formulate the propagation of multiple flows of pedestrians targeting different destinations or exits. The notations used for the potentials of cells corresponding to each exit, the maximum flows of the arcs, and the capacities of the nodes in the evacuation network are the same as those employed in Section 2.2. Other variables are notated as follows. Let $E$ denote the set of destination nodes corresponding to the exits, $n_{i}^{e}(t)$ the number of pedestrians targeting destination node $e$ at node $i$ at time step $t$, and $D_{i j}^{e}(t)$ the proportion of pedestrians targeting destination node $e$ at node $i$ who can move to the adjacent node $j$ in interval 
$\Delta t$ between time steps $t$ and $t+1$.

For the adjacent downstream node $j, D_{i j}^{e}(t)$ is assumed to be in proportion to the product of the number of pedestrians accommodated by the empty space at node $j$ at time step $t, N_{j}(t)-\sum_{m \in E} n_{j}^{m}(t)$, and the difference between the potentials of the two nodes corresponding to destination node $e, P_{i}^{e}-P_{j}^{e}$. It follows that

$$
D_{i j}^{e}(t)=\left\{\begin{array}{cc}
\frac{\left(P_{i}^{e}-P_{j}^{e}\right)\left[N_{j}(t)-\sum_{m \in E} n_{j}^{m}(t)\right]}{\sum_{k \in R_{i}^{e}}\left\{\left(P_{i}^{e}-P_{k}^{e}\right)\left[N_{k}(t)-\sum_{m \in E} n_{k}^{m}(t)\right]\right\}}, & j \in R_{i}^{e}, \\
0, & \text { otherwise, }
\end{array}\right.
$$

where $R_{i}^{e}$ is the set of neighboring nodes downstream of node $i$ corresponding to destination node $e$.

Let $w_{i j}^{e}(t)$ be the number of pedestrians targeting destination node $e$ who can be sent to node $j$ from node $i$ in interval $\Delta t$ between time steps $t$ and $t+1$. It is assumed that the maximum number of pedestrians targeting destination node $e$ and moving to node $j$ from node $i$ in interval $\Delta t$ between time steps $t$ and $t+1$ is positively proportional to the number of pedestrians targeting destination node $e$ at node $i$ who can move to node $j$ in the interval $\Delta t . w_{i j}^{e}(t)$ is then formulated as

$$
w_{i j}^{e}(t)=\min \left\{D_{i j}^{e}(t) n_{i}^{e}(t), Q_{i j}(t) \frac{D_{i j}^{e}(t) n_{i}^{e}(t)}{\sum_{m \in E}\left[D_{i j}^{m}(t) n_{i}^{m}(t)+D_{j i}^{m}(t) n_{j}^{m}(t)\right]}\right\} .
$$

Furthermore, it is assumed that the proportion of empty space at a node that can receive pedestrians from an adjacent upstream node is partly related to the number of pedestrians who can be sent to the node from upstream nodes. The number of pedestrians targeting destination node $e$ who move from node $i$ to node $j$ in interval $\Delta t$ between time steps $t$ and $t+1, \quad y_{i j}^{e}(t)$, is then written as

$$
y_{i j}^{e}(t)=\min \left\{w_{i j}^{e}(t), \frac{N_{j}(t)-\sum_{m \in E} n_{j}^{m}(t)}{\sum_{m \in E} \sum_{k \in S_{j}^{m}} w_{k j}^{m}(t)} w_{i j}^{e}(t)\right\},
$$

where $S_{j}^{e}$ is the set of neighboring nodes upstream of node $j$ corresponding to destination node $e$.

Based on the above definitions, the propagation of multiple flows of pedestrians 
targeting different exits in the evacuation network with multiple destination nodes can be described by

$$
n_{i}^{e}(t+1)=n_{i}^{e}(t)+\sum_{k \in S_{i}^{e}} y_{k i}^{e}(t)-\sum_{j \in R_{i}^{e}} y_{i j}^{e}(t),
$$

for all nodes and time steps.

In the following section, we apply the propagation model to a simulation scenario involving multiple exits.

\subsection{Numerical Simulations}

The propagation model for multiple flows of pedestrians is used to simulate pedestrian evacuation in the scenario shown in Fig. 4 (c). The indoor areas are discretized into $19 \times 6+20 \times 5$ cells, there are no obstacles in this scenario, and the four walls each house one exit. The following parameters take values the same as those used in the numerical simulations presented in Section 2.3. In other words, the time interval $\Delta t=1.2$, the width of the cells $s=1.0$, the maximum number of pedestrians passing through the opening between two cells in a time interval $\bar{Q}=10$, the maximum capacity of an empty cell to accommodate pedestrians $\bar{N}=16$, and the maximum number of pedestrians moving to each destination node $j_{0}$ from upstream linked node $i_{0}$ in each time interval $Q_{i_{0} j_{0}}(t)=15$.

Fig. 9 displays pseudo-color plots delineating the ratio of the total number of pedestrians in each cell to the capacity of the cell in scenario (c) at time steps 10, 40, and 80 in the simulation. Here, the ratio of the number of pedestrians targeting each of the four exits in each cell to the capacity of the cell at the initial time is 0.15 and the parameter $\theta$ in the algorithm used to compute the potentials is 0.9. Pedestrians targeting different exits affect each other and a number of pedestrians slow down due to confronting others targeting the other exits. As a result, crowds appear not only near the four exits, but also at the center of the room. Near each exit, pedestrians targeting the exit compete with each other for the limited space available to go through the exit. However, at the center of the room, pedestrians targeting different exits share the paths to each exit.

The simulation results reveal the collective behavior of pedestrians targeting the same 
exit. Fig. 10 shows pseudo-color plots delineating the ratio of the number of pedestrians targeting exit 1 in each cell to the capacity of the cell at time steps 10, 40, and 70 in this scenario. Comparison of Figs. 5 and 10 shows that for the same $\theta$ value of 0.9, the crowd near the exit in the north wall takes a different shape in each of the two scenarios. This is understandable due to pedestrians on vertical paths approaching the exit at a slower speed than those on horizontal paths in scenario (c), leading to the horizontal dimension of the crowd being larger than the vertical dimension.

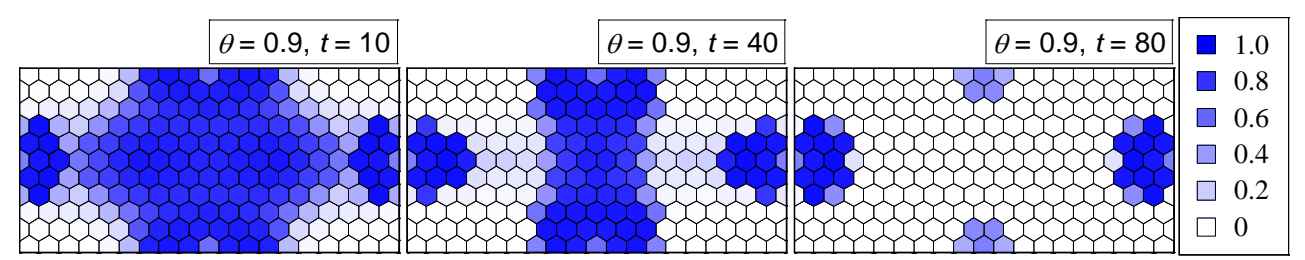

Fig. 9. Pseudo-color plots delineating the ratio of the total number of pedestrians in each cell to the capacity of the cell at time steps 10,40 , and 80 during an evacuation process in scenario (c) when $n_{i}^{e}(0)=0.15 N_{i}(0)$ (where $i$ is any node and $e \in E$ ) and the parameter $\theta=0.9$.

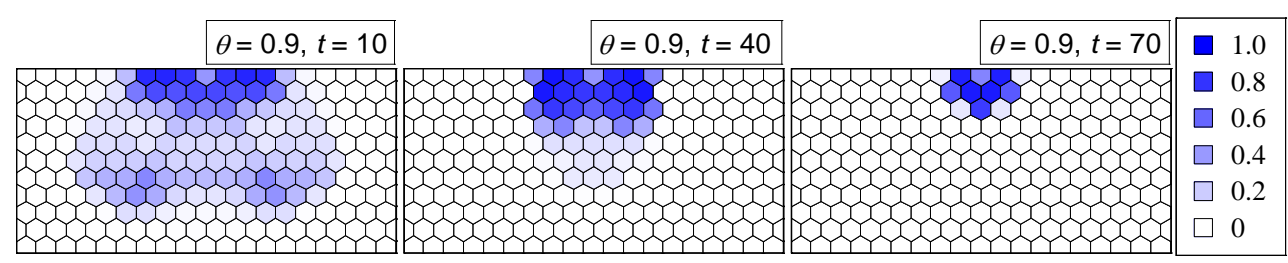

Fig. 10. Pseudo-color plots delineating the ratio of the number of pedestrians targeting exit 1 in each cell to the capacity of the cell at time steps 10,40, and 70 during an evacuation process in scenario (c) when $n_{i}^{e}(0)=0.15 N_{i}(0)$ (where $i$ is any node and $e \in E$ ) and the parameter $\theta=0.9$.

The spillback and dissipation of pedestrians in this scenario are also investigated. Fig. 11 shows time-space diagrams delineating the ratio of the total number of pedestrians in each cell to the capacity of the cell on lines 9-12 (see Fig. 4). One can see that on line 9, two transition zones in which the pedestrian density level is relatively low emerge and become 
larger as time elapses over the course of the evacuation process. As a result, the pedestrian queue on the line is divided into three short queues, two near each of the two exits and one in the middle of the line. When the queue in the middle of the line disappears, the two transition zones also disappear. For the other three lines, the three phases described in Section 2.3 can also be observed. Furthermore, the three phases become more obvious on the line adjacent to exit 1 .
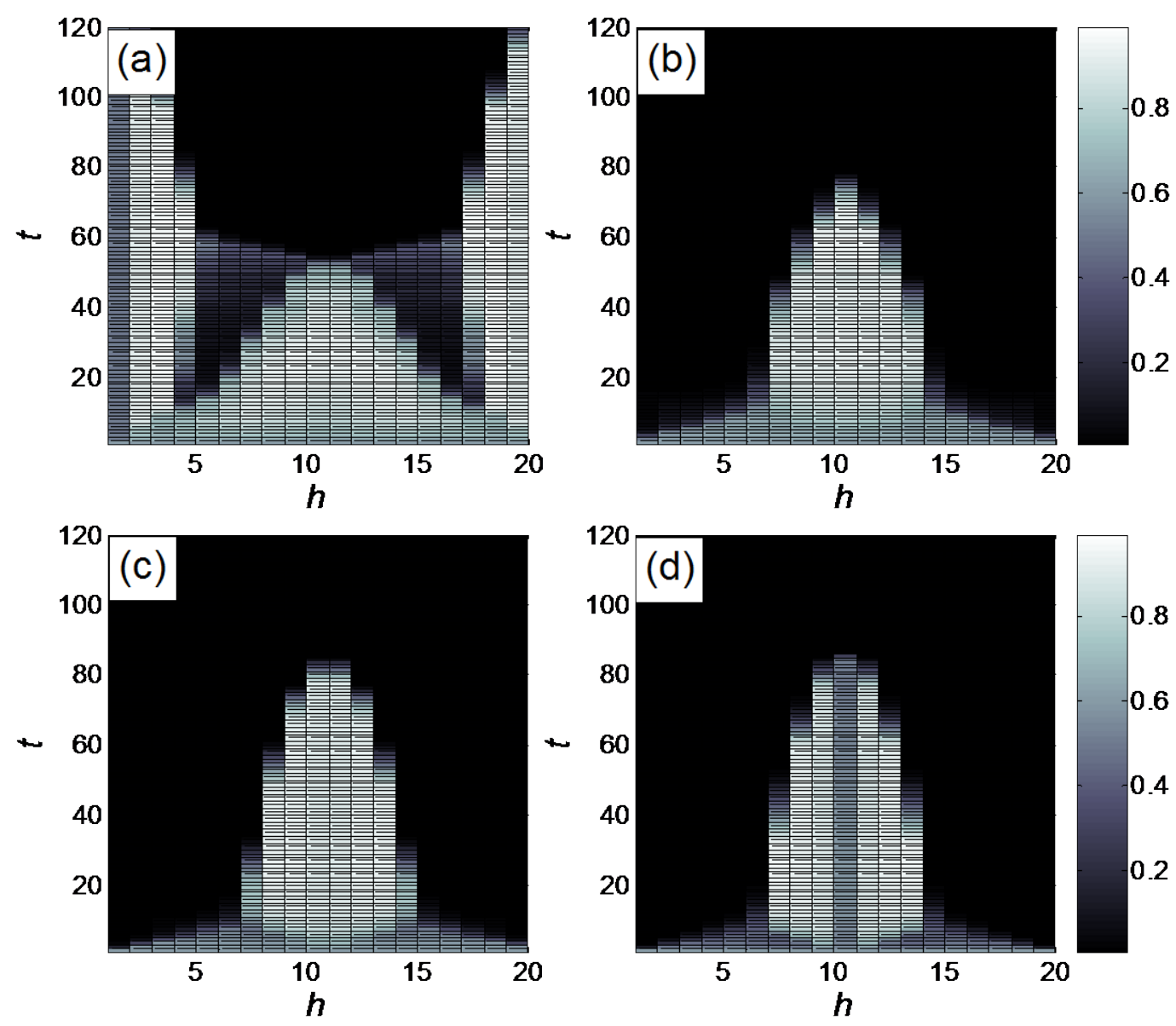

Fig. 11. Time-space diagrams delineating the ratio of the total number of pedestrians in each cell to the capacity of the cell on lines (a) 9, (b) 10, (c) 11, and (d) 12 in Fig. 4 when $\boldsymbol{\theta}=\mathbf{0 . 9}$

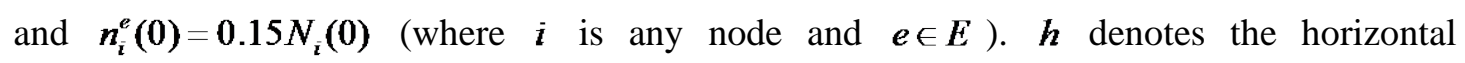
coordinates of the cells on lines 9-12.

\section{Potential considering pedestrian congestion}

\subsection{Computation process}


The model proposed in Section 2 can be used to provide valuable information for designing and adjusting the internal layout of and the positions of exits in buildings. For a building with an existing layout of internal obstacles and exit positions, another model is required to assign pedestrian flow and determine pedestrian movement routes within the building space. In dynamic vehicle traffic assignment, it is generally assumed that vehicles choose travel routes on the basis of their comparative time-varying travel costs. Travel cost consists of free-flow cost and the cost due to vehicle congestion on the route. It is computed in a nested form (Huang and Lam, 2002) and the computational process is relatively complex. Here, the generalized potential of each cell is defined and used to measure the value of the respective weightings assigned to the route distance from the cell to the target exits and the congestion disutility caused by front pedestrians on the exit routes. Suppose all pedestrians select target cells at the next time step to reduce the generalized potential of each such cell toward the destination. This implies that in selecting movement routes to target exits, pedestrians consider not only route distances to different target exits, but also take into account the congestion costs caused by front pedestrians on such routes (Hoogendoorn and Bovy, 2004a,b; Kretz, 2009; Xia et al., 2009; Hartmann, 2010).

An algorithm for computing the generalized potentials of cells is developed. Some of the notations for the algorithm are given as follows. Let $C$ denote the set of cells in the entire pedestrian space, $C^{e}$ the set of cells near exit $e, n_{i}$ the number of pedestrians in cell $i$, and $\tilde{P}_{i}^{e}$ the generalized potential of cell $i$ toward exit $e$. Let $U_{i}^{e}$ represent whether cell $i$ is assigned the generalized potential toward exit $e$. It takes the value of 0 if cell $i$ is not assigned the potential; 1 if cell $i$ will be assigned the potential; and 2 if cell $i$ has been assigned the potential. Let $\bar{m}$ denote the number of cells that will be assigned the potential. The generalized potentials are computed by the following algorithm.

Step 1: For all cells $i \in C \backslash C^{e}$, set $U_{i}^{e}=0$; for all cells $j \in C^{e}$, set $U_{j}^{e}=1$ and $\tilde{P}_{j}^{e}=1$. Set $\bar{m}=\left|C^{e}\right|$ and $l=1$;

Step 2: If $\bar{m}=0$, stop; otherwise go to step 3;

Step 3: For each cell $i \in V^{e}$, set $U_{i}^{e}=2$, where $V^{e}=\left\{i \mid i \in C, U_{i}^{e}=1\right.$ and $\left.\tilde{P}_{i}^{e} \leq l\right\}$. Set $\bar{m} \leftarrow \bar{m}-\left|V^{e}\right| ;$ 
Step 4: For each cell $i \in C$ with the $U_{i}^{e}$ value updated at step 3, check its adjacent cells in all six directions: for each cell $j$ that is connective with cell $i$ and $U_{j}^{e}=0$, set $U_{j}^{e}=1, \quad \bar{m} \leftarrow \bar{m}+1$ and, if $\left|\Psi_{j}^{e}\right|=1$, let $\tilde{P}_{j}^{e}=\tilde{P}_{i}^{e}+1+\tau n_{j} ; \quad$ if $\left|\Psi_{j}^{e}\right|>1$, let $\tilde{P}_{j}^{e}=\sum_{k \in \Psi_{j}^{e}} \tilde{P}_{k}^{e} /\left|\Psi_{j}^{e}\right|+\theta+\tau n_{j}$. Here, $\Psi_{j}^{e}=\left\{i \mid\right.$ cells $i$ and $j$ are connective, and $\left.U_{i}^{e}=2\right\}$, $\theta \in(0,1]$, and $\tau \geq 0$;

Step 5: Set $l \leftarrow l+1$ and go to step 2 .

In the algorithm, parameter $\theta$ has the same meaning as in the algorithm presented in Section 2.1 and the meaning of set $\Psi_{j}^{e}$ is similar to that of set $\Omega_{j}^{e}$. Parameter $\tau$ is used to weight the effect of the number of front pedestrians on exit routes on the potential value of a cell. A larger $\tau$ value indicates that pedestrians pay more attention to the congestion disutility caused by front pedestrians on exit routes. In the following section, we use the algorithm developed to investigate pedestrian route choice behavior taking congestion disutility into account.

\subsection{Numerical simulations}

The algorithm used to compute generalized potential is applied to the simulation scenario shown in Fig. 4 (d). The indoor areas are discretized into $19 \times 6+20 \times 5$ cells, there are two obstacles in the scenario, and one exit is located in the north wall. The input parameters take the same values as those used in previous numerical simulations, i.e., the time interval $\Delta t=1.2$, the width of the cells $s=1.0$, the maximum number of pedestrians passing through the opening between two cells in a time interval $\bar{Q}=10$, the maximum capacity of an empty cell to accommodate pedestrians $\bar{N}=16$, and the maximum number of pedestrians moving from upstream linked node $i_{0}$ to the destination node $j_{0}$ in each time interval $Q_{i_{0} j_{0}}(t)=15$.

We now investigate how congestion disutility on front routes taken by pedestrians affects their route choice behavior. The ratio of the number of pedestrians in each cell to the capacity of the cell at the initial time is shown in Fig. 12 and $\theta$ in the algorithm takes the value of 0.9. Fig. 13 shows pseudo-color plots delineating the ratio of the number of 
pedestrians in each cell to the capacity of the cell at time step 20 where the value of $\tau$ varies from 0 to 0.08 with an interval of 0.01 .

In this scenario, there are two routes approaching the exit, i.e., routes 1 and 2, running from the west and the east, respectively (see Fig. 4). At the initial time, most pedestrians congregate in cells on route 2 . Fig. 13 shows that with an increase in $\tau$, the number of pedestrians selecting route 1 increases and the number of pedestrians in the crowd on route 2 declines. This phenomenon is analyzed further through Fig. 14.

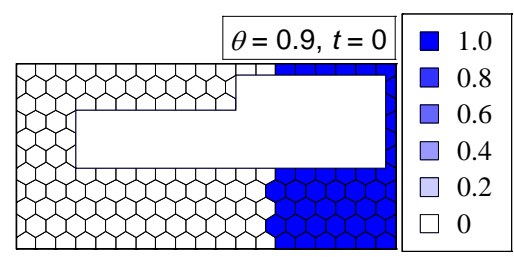

Fig. 12. A class of initial pedestrian distributions in scenario (d) in Fig. 4.
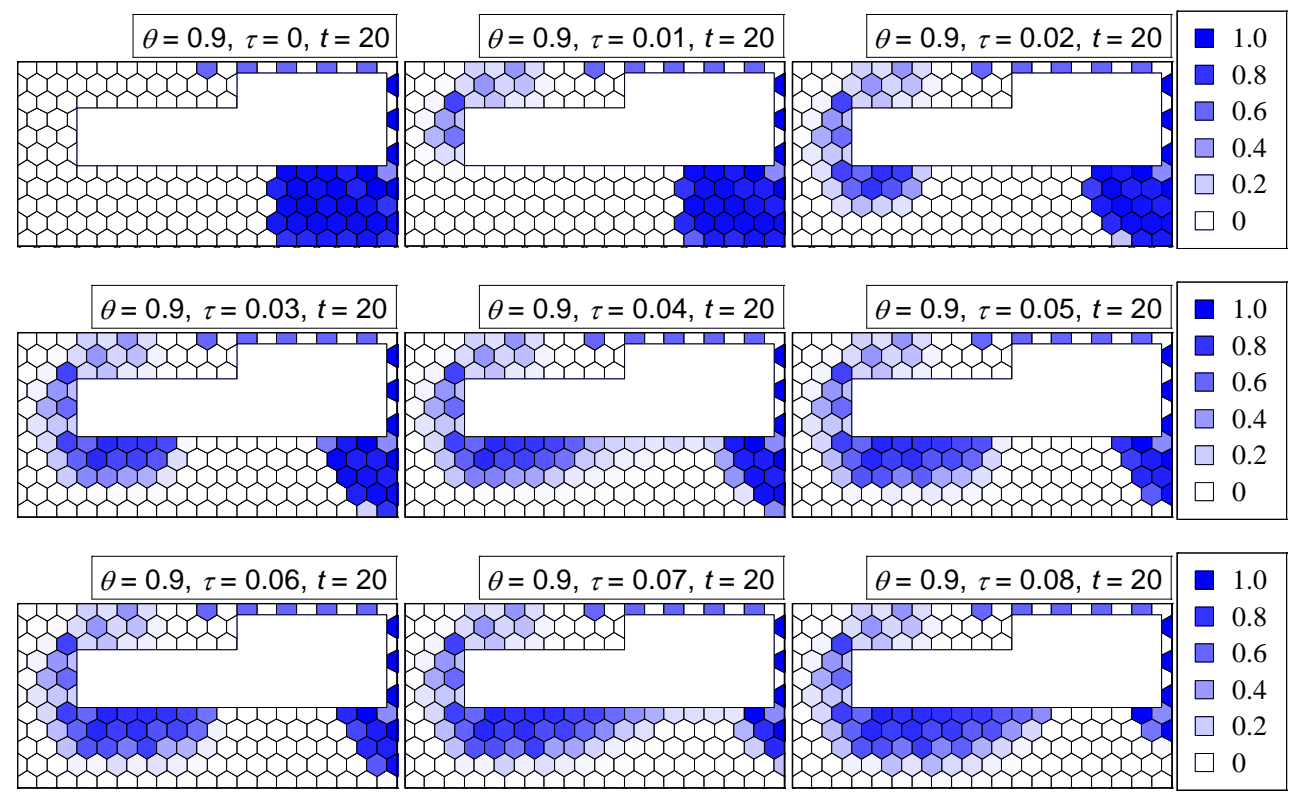

Fig. 13. Pseudo-color plots delineating the ratio of the number of pedestrians in each cell to the capacity of the cell at time step 20 during an evacuation process in scenario (d) of Fig. 4 when $\theta$ takes the value of 0.9 and $\tau$ varies from 0 to 0.08 with an interval of 0.01 . The ratio of the number of pedestrians in each cell to the capacity of the cell at the initial time is shown in Fig. 12. 
Fig. 14 displays the evolutionary process of the number of pedestrians selecting routes 1 and 2, respectively, in the above simulations. One can see that as the $\tau$ value increases, the number of pedestrians selecting route 1 increases and the time taken for all pedestrians to leave the room falls. In addition, an increasing $\tau$ value affects the rate of decline in the number of pedestrians on route 2 . When the $\tau$ value is larger than 0.05 , the time taken for all pedestrians to leave the room via each of the two routes is virtually the same and a state of equilibrium is reached.
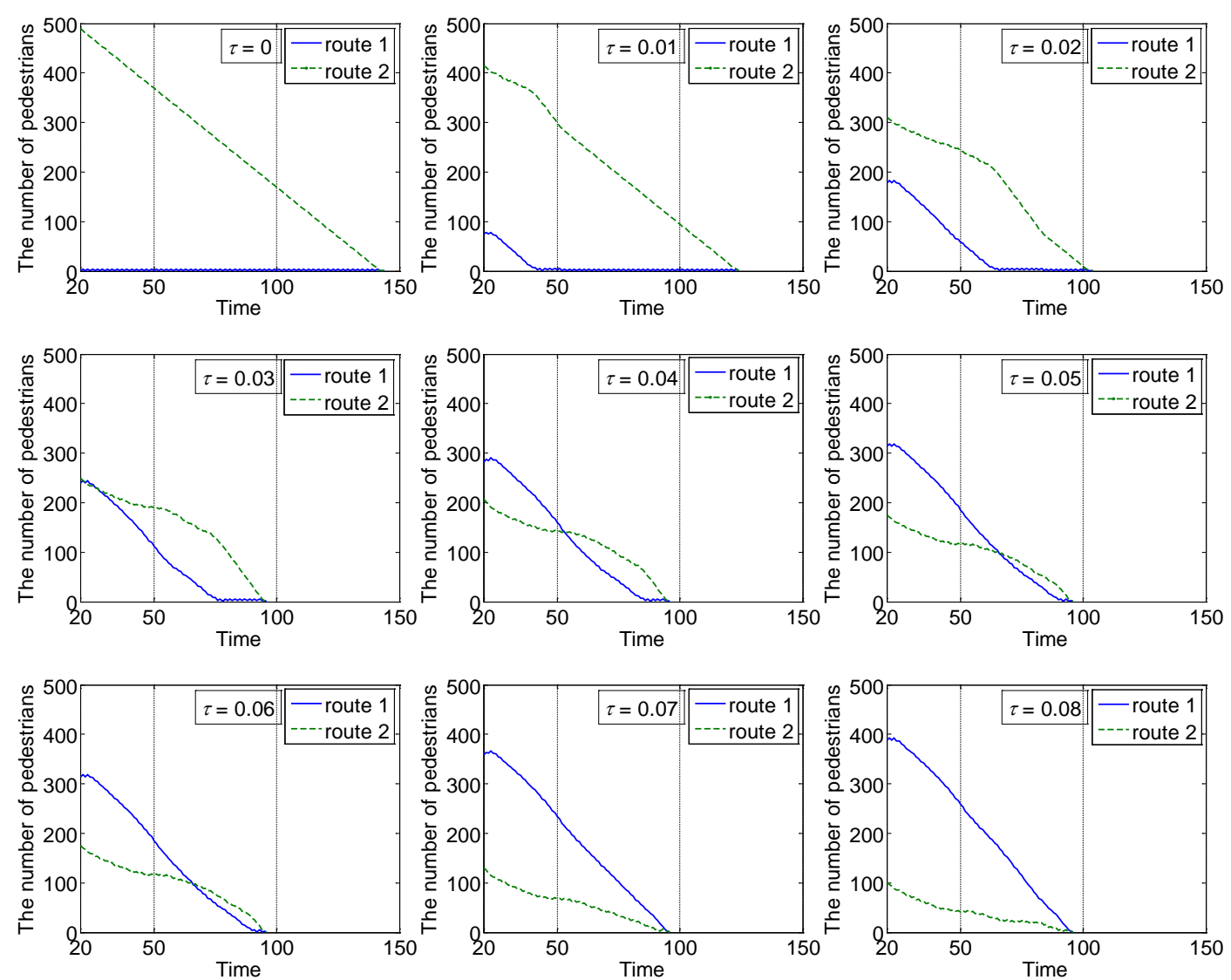

Fig. 14. The evolutionary process of the number of pedestrians selecting routes 1 and 2, respectively, in each simulation in scenario (d) of Fig. 4 when $\theta$ takes the value of 0.9 and $\tau$ varies from 0 to 0.08 with an interval of 0.01 . The ratio of the number of pedestrians in each cell to the capacity of the cell at the initial time is shown in Fig. 12.

In the final part of the analysis, we investigate the spillback and dissipation of pedestrians on lines 13 and 14 (see Fig. 4). Fig. 15 shows time-space diagrams delineating the 
ratio of the number of pedestrians in each cell to the capacity of the cell on lines 13 and 14 when $\boldsymbol{\theta}=\mathbf{0 . 9}, \boldsymbol{\tau}=\mathbf{0 . 0 6}$, and the ratio of the number of pedestrians in each cell to the capacity of the cell at the initial time is the same as that shown in Fig. 12. It can be seen that the evolution of the pedestrian flow on line 13 over time is divided into two phases. In phase one, the dissipation and propagation of the pedestrian flow occur simultaneously and a congested group of pedestrians splits into two groups with mixed flows on routes 1 and 2. In phase two, the two groups of pedestrians with mixed flows gradually dissipate. The evolution of the pedestrian flow on line 14 over time is divided into four phases. Two pedestrian flows emerge in the first two phases, the first being a relatively dense group of pedestrians that dissipates and the second being a relatively low-density group that propagates. In phase three, the overall pedestrian flow continues to dissipate and propagate simultaneously and the two groups of mixed flows on routes 1 and 2 converge into one congested flow. The congested flow gradually dissipates in phase four.
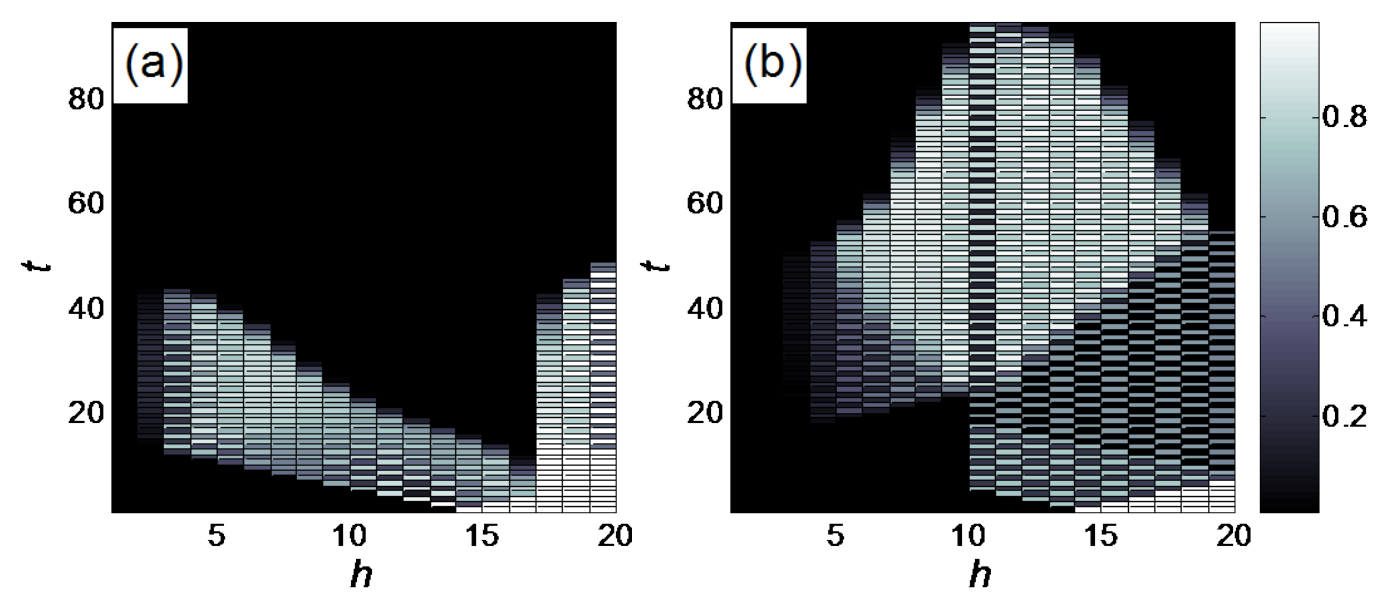

Fig. 15. Time-space diagrams delineating the ratio of the number of pedestrians in each cell to the capacity of the cell on lines (a) 13, and (b) 14 in Fig. 4 when $\boldsymbol{\theta}=\mathbf{0 . 9}, \boldsymbol{\tau}=\mathbf{0 . 0 6}$, and the ratio of the number of pedestrians in each cell to the capacity of the cell at the initial time is the same as that shown in Fig. 12. $h$ denotes the horizontal coordinates of the cells on lines 13 and 14.

\section{Conclusions}


This paper presents an innovative method of constructing a plausible framework to study pedestrian movement route choice behavior and predict the physical congestion of pedestrians attempting to evacuate from a closed area with internal obstacles. This is achieved by converting the pedestrian evacuation problem into a network problem and applying the cell transmission model proposed to determine the propagation of pedestrian flow. In this way, most current dynamic traffic assignment techniques can be used directly to solve the pedestrian evacuation problem. The proposed method is extended to examine the collection, dissipation, and propagation behavior of multiple flows of pedestrians targeting different exits and to assign pedestrian flow in space during the evacuation process.

The network-based model proposed in this paper differs from traditional network-based models in several aspects. First, because our model discretizes each room, lobby, and corridor in detail and uses multiple nodes to represent them, it allows for careful consideration of the internal obstacles in a building and the physical congestion of pedestrians. Second, the division of pedestrian space into isotropic hexagonal cells allows for the flow of pedestrians into neighboring cells to be defined in identical terms. Third, a novel feature of the model lies in the use of potential to determine route choice behavior among pedestrians. We developed two algorithms to compute the two classes of potential. One class measures the route distance to the target exit and the other reflects the value of the respective weightings assigned to the route distance to the target exit and the congested disutility caused by front pedestrians on exit routes.

Hoogendoorn and Bovy (2004a, 2004b) propose a pedestrian model based on the concept of utility maximization that can be used to predict the route choices and activity scheduling of pedestrians. They developed an approach for the dynamic assignment of pedestrian flows in continuous time and space. Our model differs from theirs on several counts. First, their model treats pedestrians as discrete individuals, whereas pedestrians in a certain area can be regarded as an entity under our model. Second, their model relies on the optimal control theory in depicting the route choices and activity scheduling of pedestrians. In contrast, our model determines pedestrian route choice on the basis of potential, a measure reflecting both the route distance to the destination and pedestrian congestion. Third, the two 
models are designed to serve different purposes. Their model is mainly used to assign pedestrian flow and predict route choice and activity scheduling among pedestrians, whereas the main application of our model is to investigate the collection, spillback, and dissipation behavior of pedestrians and assign pedestrian flow in space.

The simulation results for the four scenarios examined in this paper show that the proposed method can be used to predict the evolution of pedestrian flow over time and space in indoor areas with internal obstacles. It can also be used to model collection, propagation, and dissipation behavior among pedestrians, and in particular to evaluate the shape and extent of congestion near exits. This allows for bottlenecks caused by obstacles on an evacuation route to be identified and for propagation-dissipation processes to be explored.

Issues worthy of investigation in future research aimed at extending the method proposed in this paper include how the pedestrian evacuation process unfolds when conditions in indoor areas (e.g., the maximum number of pedestrians allowed in each cell and the maximum flow of pedestrians between cells) change over time and space. In addition, the proposed method could also be applied in studies of the emergence of collective states in systems such as flocks of birds, schools of fish, and swarms of locusts.

\section{Appendix}

\section{Model Properties}

The pedestrian propagation model discussed in Section 2.2 has the following properties.

Property 1. When $\sum_{k \in S_{j}} w_{k j}(t) \leq N_{j}(t)-n_{j}(t)$, then $N_{j}(t)-n_{j}(t) \geq \sum_{k \in S_{j}} y_{k j}(t)$ holds; when $\sum_{k \in S_{j}} w_{k j}(t)>N_{j}(t)-n_{j}(t)$, then $N_{j}(t)-n_{j}(t)=\sum_{k \in S_{j}} y_{k j}(t)$ holds.

$$
\begin{aligned}
& \text { Proof. } \sum_{k \in S_{j}} w_{k j}(t) \leq N_{j}(t)-n_{j}(t) \Rightarrow \sum_{k \in S_{j}} y_{k j}(t)=\sum_{k \in S_{j}} w_{k j}(t) \leq N_{j}(t)-n_{j}(t) . \\
& \sum_{k \in S_{j}} w_{k j}(t)>N_{j}(t)-n_{j}(t) \Rightarrow \sum_{k \in S_{j}} y_{k j}(t)=\sum_{k \in S_{j}}\left[\frac{N_{j}(t)-n_{j}(t)}{\sum_{i \in S_{j}} w_{i j}(t)} w_{k j}(t)\right]=N_{j}(t)-n_{j}(t) .
\end{aligned}
$$

Property 1 indicates that when the empty space at a node at a given time step can accommodate all of the pedestrians sent from adjacent upstream nodes in the interval between 
that time step and the next time step, the number of pedestrians accommodated by the empty space at the node at that time step is not less than the number of pedestrians moving into the node from upstream nodes in the time interval. When these empty spaces cannot accommodate these pedestrians, the two numbers of pedestrians are identical.

Property 2. The relation $n_{i}(t) \geq \sum_{j \in R_{i}} y_{i j}(t)$ holds; furthermore, when $\exists j \in R_{i}$, s.t. $\sum_{k \in S_{j}} w_{k j}(t)>N_{j}(t)-n_{j}(t)$, then $n_{i}(t)>\sum_{k \in R_{i}} y_{i k}(t)$ holds.

Proof. $\sum_{j \in R_{i}} y_{i j}(t) \leq \sum_{j \in R_{i}} w_{i j}(t) \leq \sum_{j \in R_{i}}\left[D_{i j}(t) n_{i}(t)\right]=n_{i}(t) \sum_{j \in R_{i}} D_{i j}(t)=n_{i}(t)$.

$\sum_{k \in S_{j}} w_{k j}(t)>N_{j}(t)-n_{j}(t) \Rightarrow y_{i j}(t)<w_{i j}(t)$.

In similar fashion, $\sum_{k \in R_{i}} y_{i k}(t)<n_{i}(t)$

Property 2 means that the number of pedestrians at a node at a given time step is not less than the number of pedestrians moving away from the node to downstream nodes in the interval between that time step and the next time step. Furthermore, when there is at least one node downstream of the node with empty space at a given time step that cannot accommodate all of the pedestrians sent from adjacent upstream nodes in the time interval, the former number is greater than the latter number.

Property 3. The equation $\sum_{i \in \bar{C}} n_{i}(t+1)=\sum_{i \in \bar{C}} n_{i}(t)$ holds, where $\bar{C}$ is the set of all nodes in the evacuation network.

Proof. $\sum_{i \in \bar{C}} n_{i}(t+1)=\sum_{i \in \bar{C}} n_{i}(t)+\sum_{i \in \bar{C}} \sum_{k \in S_{i}} y_{k i}(t)-\sum_{i \in \bar{C}} \sum_{j \in R_{i}} y_{i j}(t)=\sum_{i \in \bar{C}} n_{i}(t)$.

Property 3 shows that the number of pedestrians in the entire space is conservatively estimated in the propagation process.

Property 4. When $\sum_{k \in S_{j}} w_{k j}(t) \leq N_{j}(t)-n_{j}(t)$, then $n_{j}(t+1) \leq N_{j}(t)-\sum_{k \in R_{j}} y_{j k}(t)$ holds; when $\sum_{k \in S_{j}} w_{k j}(t)>N_{j}(t)-n_{j}(t)$, then $n_{j}(t+1)=N_{j}(t)-\sum_{k \in R_{j}} y_{j k}(t)$ holds.

Proof. These two results are directly deduced from Eq. (7) and Property 1.

Property 4 indicates that when the empty space at a node at a given time step can accommodate all of the pedestrians sent from adjacent upstream nodes in the interval between that time step and the next time step, the number of pedestrians at the node at the next time 
step is not more than the difference between the maximum number of pedestrians at the node at the initial time step and the number of pedestrians moving away from the node to downstream nodes in the time interval. When these empty spaces cannot accommodate these pedestrians, the number of pedestrians is equal to this difference.

Property 5. The relation $n_{i}(t+1) \geq \sum_{k \in S_{i}} y_{k i}(t)$ holds. Furthermore, when $\exists j \in R_{i}$, s.t. $\sum_{k \in S_{j}} w_{k j}(t)>N_{j}(t)-n_{j}(t)$, then $n_{i}(t+1)>\sum_{k \in S_{i}} y_{k i}(t)$ holds.

Proof. These results are directly deduced from Eq. (7) and Property 2.

Property 5 means that the number of pedestrians at a node at a given time step is not less than the number of pedestrians moving to the node from upstream nodes in the interval between the previous time step and that time step. Furthermore, when there is at least one node downstream of the node with empty space at that time step that cannot accommodate all of the pedestrians sent from adjacent upstream nodes in the time interval, the former number is greater than the latter number.

Property 6. When $n_{i}(t) \geq 0$ and $\forall j \in R_{i}, N_{j}(t) \geq n_{j}(t)$, then $n_{i}(t+1) \leq N_{i}(t)$ holds.

Proof. $\quad n_{i}(t) \geq 0$ and $\forall j \in R_{i}, \quad N_{j}(t) \geq n_{j}(t) \Rightarrow \forall j \in R_{i}, \quad y_{i j}(t) \geq 0 \Rightarrow$ $N_{i}(t)-\sum_{j \in R_{i}} y_{i j}(t) \leq N_{i}(t)$. By Property 4, $n_{i}(t+1) \leq N_{i}(t)$ holds

Property 7. When $\forall k \in S_{i}, \quad n_{k}(t) \geq 0$ and $\forall j \in R_{k}, \quad N_{j}(t) \geq n_{j}(t)$, then $n_{i}(t+1) \geq 0$ holds

Proof. $\forall k \in S_{i}, n_{k}(t) \geq 0$ and $\forall j \in R_{k}, \quad N_{j}(t) \geq n_{j}(t) \Rightarrow \forall k \in S_{i}, \quad y_{k i}(t) \geq 0$. By Property $5, \quad n_{i}(t+1) \geq 0$ holds.

Properties 6 and 7 illustrate that if the number of pedestrians at any one node at a given time step is within a certain range, then the number of pedestrians at any one node in the subsequent time step is guaranteed to remain within that range.

Empirical studies show that as pedestrian density increases, pedestrian flow increases from zero to over capacity and then returns to near zero. Clearly, this means a " $\Lambda$ " flow-density relationship exists (Virkler and Elayadath, 1994; Daamen and Hoogendoorn, 
2007). We now verify whether the model is consistent with a " $\Lambda$ " flow-density relationship. The flow-density relationship formulated by the model for the corridor shown in Fig. 16 is then investigated. In the corridor discretized into $4 \times 2+5 \times 3$ cells, pedestrians move from left to right. Let the set of neighboring cells upstream of the opening $o$ denoted by a zigzag line be $\Phi$ and the set of downstream neighboring cells be $\Omega$. The potential and maximum capacity of the cells in sets $\Phi$ and $\Omega$ and the number of pedestrians in these cells at time step $t$ are shown in Fig. 16. The maximum number of pedestrians moving into any one cell $j \in \Omega$ from another cell $i \in \Phi \cap S_{j}$ in the interval $\Delta t$ between time steps $t$ and $t+1$ is $Q_{i j}(t)=Q$.

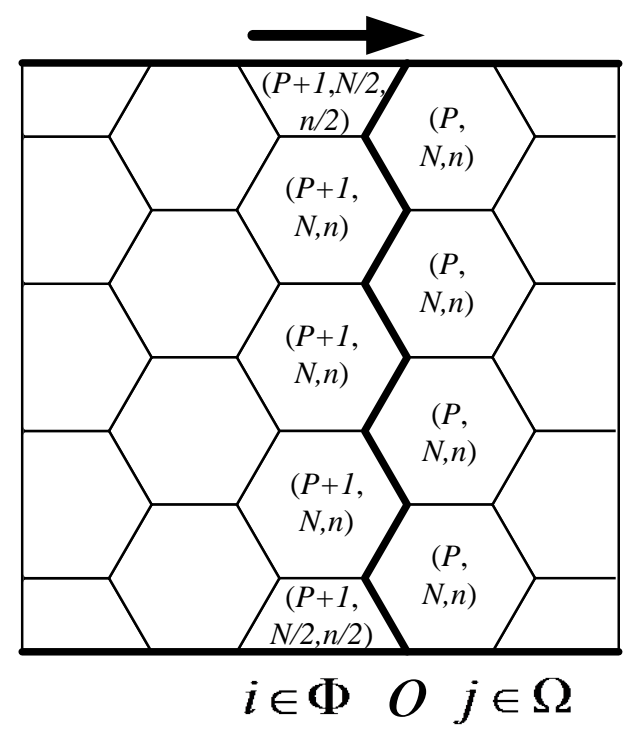

Fig. 16. A corridor discretized into $4 \times 2+5 \times 3$ cells. The direction of the arrow indicates the movement direction of pedestrians. The three numbers in each pair of brackets denote the potential and maximal capacity of the corresponding cell and the number of pedestrians in the cell at time step $t$, respectively.

The following equation can be deduced from Eqs. (3) and (4).

$$
w_{i j}(t)=\min \{n / 2, Q\}, \forall j \in \Omega \text {, and } i \in \Phi \cap S_{j} .
$$

Thus, the number of pedestrians moving into cell $j \in \Omega$ from cell $i \in \Phi \cap S_{j}$ in the interval $\Delta t$ between time steps $t$ and $t+1, y_{i j}(t)$, is written as 


$$
y_{i j}(t)=\min \{n / 2, Q,(N-n) / 2\}, j \in \Omega \text {, and } i \in \Phi \bigcap S_{j} .
$$

Let the ratio of the total number of pedestrians in the region covering all of the cells in sets $\Phi$ and $\Omega$ at time $t$ to the capacity of the region be $\rho$, i.e., $\rho=n / N$. Let the number of pedestrians passing through the opening $o$ in the interval $\Delta t$ between time steps $t$ and $t+1$ be $\Gamma(\rho)$. Clearly, we have

$$
\Gamma(\rho)=\min \{4 N \rho, 8 Q, 4 N(1-\rho)\} .
$$

The model is thus consistent with a " $\Lambda$ " flow-density relationship.

\section{Acknowledgements}

We extend our sincere thanks to the anonymous referees for their constructive comments. The work described in this paper was jointly supported by grants from the National Natural Science Foundation of China (70821061, 71001047), the National Basic Research Program of China (2006CB705503), the Program of Higher-level Talents of Inner Mongolia University (Z20090113), and the Research Grants Council of the Hong Kong Special Administrative Region, China (HKU7184/10E).

\section{References}

Asano, M., Sumalee, A., Kuwahara, M., Tanaka, S., 2007. Dynamic cell transmission-based pedestrian model with multidirectional flows and strategic route choices. Transportation Research Record 2039, 42-49.

Burstedde, C., Klauck, K., Schadschneider, A., Zittartz, J., 2001. Simulation of pedestrian dynamics using a two-dimensional cellular automaton. Physica A 295 (3-4), 507-525.

Cepolina, E.M., 2005. A methodology for defining building evacuation routes. Civil Engineering and Environmental Systems 22 (1), 29-47.

Cepolina, E.M., 2009. Phased evacuation: an optimisation model which takes into account the capacity drop phenomenon in pedestrian flows. Fire Safety Journal 44 (4), 532-544.

Cervero, R., Sarmiento, O.L., Jacoby, E., Gomez, L.F., Neiman, A., 2009. Influences of built environments on walking and cycling: lessons from Bogotá. International Journal of 
Sustainable Transportation 3 (4), 203-226.

Chen, P.H., Feng, F., 2009. A fast flow control algorithm for real-time emergency evacuation in large indoor areas. Fire Safety Journal 44 (5), 732-740.

Couzin, I.D., Krause, J., Franks, N.R., Levin, S.A., 2005. Effective leadership and decision-making in animal groups on the move. Nature 433 (3), 513-516.

Couzin, I.D., Krause, J., James, R., Ruxton, G.D., Franks, N.R., 2002. Collective memory and spatial sorting in animal groups. Journal of Theoretical Biology 218 (1), 1-11.

Cucker, F., Smale, S., 2007. Emergent behavior in flocks. IEEE Transactions on Automatic Control 52 (5), 852-862.

Daamen, W., Hoogendoorn, S.P., 2007. Flow-density relations for pedestrian traffic. In: Traffic and Granular Flow’05. Springer, Berlin, Heidelberg, pp. 315-322.

Daganzo, C.F., 1994. The cell transmission model: a simple dynamic representation of highway traffic. Transportation Research Part B 28 (4), 269-287.

Daganzo, C.F., 1995. The cell transmission model part II: network traffic. Transportation Research Part B 29 (2), 79-93.

Guo, R.Y., Huang, H.J., 2010. Logit-based exit choice model of evacuation in rooms with internal obstacles and multiple exits. Chinese Physics B 19 (3), 030501.

Guo, R.Y., Wong, S.C., Huang, H.J., Zhang, P., Lam, W.H.K., 2010. A microscopic pedestrian-simulation model and its application to intersecting flows. Physica A 389 (3), 515-526.

Hartmann, D., 2010. Adaptive pedestrian dynamics based on geodesics. New Journal of Physics 12, 043032.

Helbing, D., Farkas, I., Vicsek, T., 2000. Simulating dynamical features of escape panic. Nature 407 (28), 487-490.

Helbing, D., Molnár, P., 1995. Social force model for pedestrian dynamics. Physical Review E 51 (5), 4282-4286.

Hill, M.R., 1982. Spatial structure and decision-making of pedestrian route selection through an urban environment. Ph.D. thesis, University Microfilms International.

Hoogendoorn, S.P., Bovy, P.H.L., 2004a. Pedestrian route-choice and activity scheduling 
theory and models. Transportation Research Part B 38 (2), 169-190.

Hoogendoorn, S.P., Bovy, P.H.L., 2004b. Dynamic user-optimal assignment in continuous time and space. Transportation Research Part B 38 (7), 571-592.

Huang, H.J., Guo, R.Y., 2008. Static floor field and exit choice for pedestrian evacuation in rooms with internal obstacles and multiple exits. Physical Review E 78 (2), 021131.

Huang, H.J., Lam, W.H.K., 2002. Modeling and solving the dynamic user equilibrium route and departure time choice problem in network with queues. Transportation Research Part B 36 (3), 253-273.

Huang, L., Wong, S.C., Zhang, M., Shu, C.W., Lam, W.H.K., 2009. Revisiting Hughes' dynamic continuum model for pedestrian flow and the development of an efficient solution algorithm. Transportation Research Part B 43 (1), 127-141.

Hughes, R.L., 2002. A continuum theory for the flow of pedestrians. Transportation Research Part B 36 (6), 507-535.

Kirchner, A., Schadschneider, A., 2002. Simulation of evacuation processes using a bionics-inspired cellular automaton model for pedestrian dynamics. Physica A 312 (1-2), 260-276.

Kretz, T., 2009. Pedestrian traffic: on the quickest path. Journal of Statistical Mechanics: Theory and Experiment, P03012.

Langston, P.A., Masling, R., Asmar, B.N., 2006. Crowd dynamics discrete element multi-circle model. Safety Science 44 (5), 395-417.

Lin, P., Lo, S.M., Huang, H.C., Yuen, K.K., 2008. On the use of multi-stage time-varying quickest time approach for optimization of evacuation planning. Fire Safety Journal 43 (4), 282-290.

Lo, H.K., Szeto, W.Y., 2002. A cell-based variational inequality formulation of the dynamic user optimal problem. Transportation Research Part B 36 (5), 421-443.

Parrish, J.K., Edelstein-Keshet, L., 1999. Complexity, pattern, and evolutionary trade-offs in animal aggregation. Science 284 (2), 99-101.

Pursals, S.C., Garzón, F.G., 2009. Optimal building evacuation time considering evacuation routes. European Journal of Operational Research 192 (2), 692-699. 
Senevarante, P.N., Morall, J.F., 1985. Analysis of factors affecting the choice of route of pedestrians. Transportation Planning and Technology 10(2), 147-159.

Szeto, W.Y., Lo, H.K., 2004. A cell-based simultaneous route and departure time choice model with elastic demand. Transportation Research Part B 38 (7), 593-612.

Szeto, W.Y., Lo, H.K., 2006. Dynamic traffic assignment: properties and extensions. Transportmetrica 2 (1), 31-52.

Thompson, P.A., Marchant, E.W., 1995. Computer and fluid modelling of evacuation. Safety Science 18 (4), 277-289.

Virkler, M.R., Elayadath, S., 1994. Pedestrian speed-flow-density relationships. Transportation Research Record 1438, 51-58.

Walton, D., Sunseri, S., 2010. Factors influencing the decision to drive or walk short distances to public transport facilities. International Journal of Sustainable Transportation 4 (4), 212-226.

Xia, Y., Wong, S.C., Shu, C.W., 2009. Dynamic continuum pedestrian flow model with memory effect. Physical Review E 79 (6), 066113.

Xia, Y., Wong, S.C., Zhang, M., Shu, C.W., Lam, W.H.K., 2008. An efficient discontinuous Galerkin method on triangular meshes for a pedestrian flow model. International Journal for Numerical Methods in Engineering 76 (3), 337-350.

Yuan, J.P., Fang, Z., Wang, Y.C., Lo, S.M., Wang, P., 2009. Integrated network approach of evacuation simulation for large complex buildings. Fire Safety Journal 44 (2), 266-275. 